\title{
Entropy-Corrected New Agegraphic Dark Energy Model in Hořava-Lifshitz Gravity
}

\author{
Piyali Bagchi Khatua ${ }^{1 *}$, Shuvendu Chakraborty ${ }^{2 \dagger}$ and Ujjal Debnath ${ }^{3 \ddagger}$ \\ ${ }^{1}$ Department of CSE, Netaji Subhas Engineering College, Garia, Kolkata-700 152, India. \\ ${ }^{2}$ Department of Mathematics,Seacom Engineering College, Howrah, 711 302, India. \\ ${ }^{3}$ Department of Mathematics, Bengal Engineering and Science University, Shibpur, Howrah-711 103, India.
}

(Dated: November 21, 2018)

\begin{abstract}
In this work, we have considered the entropy-corrected new agegraphic dark energy (ECNADE) model in Hořava-Lifshitz gravity in FRW universe. We have discussed the correspondence between ECNADE and other dark energy models such as DBI-essence,Yang-Mills dark energy, Chameleon field, Non-linear electrodynamics field and hessence dark energy in the context of Hořava-Lifshitz gravity and reconstructed the potentials and the dynamics of the scalar field theory which describe the ECNADE.
\end{abstract}

\section{INTRODUCTION}

Recent observational data indicates that our universe is currently under accelerating phase [1 - 3]. On the basis of this observation one can state that if Einstein's theory of gravity is acceptable on cosmological scales, then our universe must dominated by a mysterious form of energy called dark energy. Scalar field models arise in string theory and are studied as promising choices for dark energy. A lot of works dealing with scalar field dark energy models are available which influence in the acceleration of the universe [4-28]. Recently, the cosmic acceleration is understood by imposing a concept of modification of gravity for an alternative candidate of dark energy $[29,30]$. This concept provides very natural gravitational alternative for exotic matter. This type of gravity is predicted by string/M-theory. The explanation of the phantom or non-phantom or quintom phase of the universe can be described by this gravity without introducing negative kinetic term of dark energies. Recently Hořava [31 - 34] proposed a new theory of gravity. It is renormalizable with higher spatial derivatives in four dimensions which reduces to Einstein's gravity with non-vanishing cosmological constant in IR but with improved UV behaviours. It is similar to a scalar field theory of Lifshitz [35] in which the time dimension has weight 3 if a space dimension has weight 1 and this theory is called Hořava-Lifshitz gravity. Hořava-Lifshitz gravity has been studied and extended in detail and applied as a cosmological framework of the universe [36 - 38].

To understand the recent cosmic acceleration many theoretical attempts have been taken in the framework of fundamental theory such as string theory or quantum gravity. After several attempts on some basic quantum gravitational principle scientists found two important models named holographic dark energy model (HDE) and the agegraphic dark energy model (ADE). HDE and ADE are originated or motivated by holographic hypothesis [39] and uncertainty relation of quantum mechanics. From quantum field theory, it can be conclude that the ultraviolet cut-off $\Lambda$ could be related to the infrared cut-off $L$ due to the limit set by forming a black hole, obeying the fact that the quantum zero-point energy of a system with size $L$ should not exceed the mass of a black hole, i.e. $L^{3} \Lambda^{3} \leqslant\left(M_{p} L\right)^{3 / 2}[40]$. Considering the energy density corresponding to the zero point energy and cut-off $\Lambda$ as $\rho_{D} \propto \Lambda^{4}$ the previous expression becomes $L^{3} \rho_{D} \leqslant L M_{p}^{2}$. So the energy density of HDE becomes $\left(\rho_{D}=3 n^{2} M_{p}^{2} / L^{2}\right)$, where $3 n^{2}$ is a constant and attached for convenience. In HDE model the black hole entropy $S$ plays a crucial role as the HDE density depends on the entropy area relation $S \propto A \propto L^{2}$ of black holes in Einstein's gravity, where $A$ is the area of the horizon [41]. If $L$ is taken as the Hubble horizon $H^{-1}$, the observational density of the dark energy is very much comparable to $\rho_{D}[42]$. But there is a theoretical problem regarding equation of state to accelerate the universe [43].

Motivated from the thermal equilibrium fluctuations and quantum fluctuations of loop quantum gravity (LQG) one can modified this after including the logarithmic correction as [44]

\footnotetext{
* piyali.bagchi@yahoo.co.in

† shuvendu.chakraborty@gmail.com

‡ujjaldebnath@yahoo.com
} 


$$
S=\frac{A}{4 G}+\gamma \ln \frac{A}{4 G}+\beta
$$

where $\gamma$ and $\beta$ are two dimensionless constants of order unity. Using this corrected entropy area relation, the energy density of the entropy corrected HDE can be written as [45]

$$
\rho_{D}=3 n^{2} m_{p}^{2} L^{-2}+\gamma \ln \left(m_{p}^{2} L^{2}\right) L^{-4}+\beta L^{-4}
$$

For large $L$ equation (2) reduces to ordinary HDE energy density as the last two terms become negligible. So the correction make sense only at the early stage of the universe and when universe become large entropy corrected HDE reduces to ordinary HDE.

Since the origin of HDE and ADE are same, we would like to consider here the so-called Entropy Corrected New Agegraphic DE (ECNADE) model. In this model it is assumed that the observed dark energy comes from the space-time and matter field fluctuations in the universe [46, 47]. In agegraphic model, the age of the universe is chosen as the length measure, instead of horizon distance to avoid the casualty problem in holographic dark energy. Cai proposed the original ADE model to explain the accelerated expansion of the universe on the basis of two arguments- Karolyhazy relation [48] and Maziashvili arguments [49]. But the original ADE model has some difficulties to explain matter dominated epoch as the time scale is chosen as the age of the universe. Then Wei and Cai [47] consider the time scale as conformal time and propose the energy density of the NADE as $\left(\rho_{x}=3 n^{2} M_{p}^{2} / T^{2}\right)$ where $T$ is the conformal time of FRW model can be written as,

$$
T=\int \frac{d t}{a}=\int_{0}^{a} \frac{d a}{H a^{2}}
$$

where $a$ is the scale factor and $H \equiv \frac{\dot{a}}{a}$ is the Hubble parameter.

So now one can consider just replacing $L$ of the equation (2) by the conformal time $T$, the energy density of ECNADE as [50]

$$
\rho_{x}=\frac{3 n^{2} m_{p}^{2}}{T^{2}}+\frac{\gamma \ln \left(m_{p}^{2} T^{2}\right)}{T^{4}}+\frac{\beta}{T^{4}}
$$

Similar as ECHDE we see that the last two terms of the equation (4) can be comparable to the first term at the early epoch of the universe and negligible when the conformal time scale $T$ becomes large. Therefore, the entropy correction can be important at the early time and the ECNADE model as ECHDE.

In this work we discuss the correspondence between ECNADE and other dark energy models such as DBIessence, Yang-Mills dark energy, Chameleon field, Non-linear electrodynamics field and hessence dark energy in the context of Hořava-Lifshitz gravity and reconstructed the potentials and the dynamics of the scalar field theory which describe the ECNADE and support the current acceleration of the universe.

\section{HOŘAVA-LIFSHITZ GRAVITY THEORY AND INTERACTING ECNADE MODEL}

In the $(3+1)$ dimensional Arnowitt-Deser-Misner formalism the full metric is written as [51],

$$
d s^{2}=-N^{2} d t^{2}+g_{i j}\left(d x^{i}+N^{i} d t\right)\left(d x^{j}+N^{j} d t\right)
$$

Under the detailed balance condition the full action condition of Hořava-Lifshitz gravity [52] is given by,

$$
S=\int d t d^{3} x \sqrt{g} N\left[\frac{2}{\kappa^{2}}\left(K_{i j} K^{i j}-\lambda K^{2}\right)+\frac{\kappa^{2}}{2 \omega^{4}} C_{i j} C^{i j}-\frac{\kappa^{2} \mu \epsilon^{i j k}}{2 \omega^{2} \sqrt{g}} R_{i l} \nabla_{j} R_{k}^{l}\right.
$$




$$
\left.-\frac{\kappa^{2} \mu^{2}}{8} R_{i j} R^{i j}+\frac{\kappa^{2} \mu^{2}}{8(1-3 \lambda)}\left(\frac{1-4 \lambda}{4} R^{2}+\Lambda R-3 \Lambda^{2}\right)\right]
$$

where

$$
K_{i j}=\frac{1}{2 N}\left(\dot{g}_{i j}-\nabla_{i} N_{j}-\nabla_{j} N_{i}\right)
$$

is the extrinsic curvature and

$$
C^{i j}=\frac{\epsilon^{i k l}}{\sqrt{g}} \nabla_{k}\left(R_{i}^{j}-\frac{1}{4} R \delta_{l}^{j}\right)
$$

is known as Cotton tensor and the covariant derivatives are defined with respect to the spatial metric $g_{i j}$. $\epsilon^{i j k}$ is the totally antisymmetric unit tensor, $\lambda$ is a dimensionless coupling constant and the variable $\kappa, \omega$ and $\mu$ are constants with mass dimensions $-1,0,1$ respectively. Also $\Lambda$ is a positive constant, which as usual is related to the cosmological constant in the IR limit.

Now, in order to focus on cosmological frameworks, we impose the so called projectability condition and use a FRW metric we get,

$$
N=1, g_{i j}=a^{2}(t) \gamma_{i j}, N^{i}=0
$$

with

$$
\gamma_{i j} d x^{i} d x^{j}=\frac{d r^{2}}{1-k r^{2}}+r^{2} d \Omega_{2}^{2}
$$

where $k=0,-1,+1$ corresponding to flat, open and closed respectively. By varying $N$ and $g_{i j}$, we obtain the non-vanishing equations of motions:

$$
H^{2}=\frac{\kappa^{2}}{6(3 \lambda-1)} \rho+\frac{\kappa^{2}}{6(3 \lambda-1)}\left[\frac{3 \kappa^{2} \mu^{2} k^{2}}{8(3 \lambda-1) a^{4}}+\frac{3 \kappa^{2} \mu^{2} \Lambda^{2}}{8(3 \lambda-1)}\right]-\frac{\kappa^{4} \mu^{2} k \Lambda}{8(3 \lambda-1)^{2} a^{2}}
$$

and

$$
\dot{H}+\frac{3}{2} H^{2}=-\frac{\kappa^{2}}{4(3 \lambda-1)} p-\frac{\kappa^{2}}{4(3 \lambda-1)}\left[\frac{\kappa^{2} \mu^{2} k^{2}}{8(3 \lambda-1) a^{4}}-\frac{3 \kappa^{2} \mu^{2} \Lambda^{2}}{8(3 \lambda-1)}\right]-\frac{\kappa^{4} \mu^{2} k \Lambda}{16(3 \lambda-1)^{2} a^{2}}
$$

The term proportional to $a^{-4}$ is the usual "dark radiation", present in Hořava-Lifshitz cosmology while the constant term is just the explicit cosmological constant. For $k=0$, there is no contribution from the higher order derivative terms in the action. However for $k \neq 0$, their higher derivative terms are significant for small volume i.e., for small $a$ and become insignificant for large $a$, where it agrees with general relativity. As a last step, requiring these expressions to coincide the standard Friedmann equations, in units where $c=1$,

$$
G_{c}=\frac{\kappa^{2}}{16 \pi(3 \lambda-1)}
$$

and

$$
\frac{\kappa^{4} \mu^{2} \Lambda}{8(3 \lambda-1)^{2}}=1
$$

where $G_{c}$ is the "cosmological" Newton's constant. We mention that in theories with Lorentz invariance breaking (such is Hořava-Lifshitz one) the "gravitational" Newton's constant $G$, that is the one that is present 
in the gravitational action, does not coincide with $G_{c}$, that is the one that is present in Friedmann equations, where

$$
G=\frac{\kappa^{2}}{32 \pi}
$$

as it can be straightforwardly read from the action. In the $\operatorname{IR}(\lambda=1)$ where Lorentz invariance is restored, $G_{c}=G$. Using the above identifications, we can re-write the Friedmann equations as,

$$
H^{2}+\frac{k}{a^{2}}=\frac{8 \pi G_{c}}{3}\left(\rho_{x}+\rho_{m}\right)+\frac{k^{2}}{2 \Lambda a^{4}}+\frac{\Lambda}{2}
$$

and

$$
\dot{H}+\frac{3}{2} H^{2}+\frac{k}{2 a^{2}}=-4 \pi G_{c}\left(p_{x}+p_{m}\right)-\frac{k^{2}}{4 \Lambda a^{4}}+\frac{3 \Lambda}{4}
$$

Here, $\rho_{x}$ and $p_{x}$ is the energy density and pressure of the dark energy, $\rho_{m}$ and $p_{m}$ is the energy density and pressure of the matter respectively and $m_{p}^{2}=\frac{1}{8 \pi G_{c}}$ is modified Plank mass. Now equation (16) can be written as,

$$
1+\frac{k}{a^{2} H^{2}}=\frac{\rho_{x}}{3 m_{p}^{2} H^{2}}+\frac{\rho_{m}}{3 m_{p}^{2} H^{2}}+\frac{k^{2}}{2 \Lambda a^{4} H^{2}}+\frac{\Lambda}{2 H^{2}}
$$

which gives,

$$
1+\Omega_{k}=\Omega_{x}+\Omega_{m}+\frac{\Omega_{k}^{2}}{2 \Omega_{\Lambda}}+\frac{\Omega_{\Lambda}}{2}
$$

where $\Omega_{k}, \Omega_{x}$ and $\Omega_{m}$ are dimensionless parameters defined as, $\Omega_{k}=\frac{k}{a^{2} H^{2}}, \Omega_{x}=\frac{\rho_{x}}{3 m_{p}^{2} H^{2}}, \quad \Omega_{\Lambda}=\frac{\Lambda}{H^{2}}$ and $\Omega_{m}=$ $\frac{\rho_{m}}{3 m_{p}^{2} H^{2}}$.

Now the energy density of the ECNADE is given in equation (4). If $\beta=0$ and $\gamma=0$ then it is same as holographic dark energy model. Now from (4) we can write,

$$
\Omega_{x}=\frac{3 n^{2} m_{p}^{2} T^{2}+\gamma \ln \left(m_{p}^{2} T^{2}\right)+\beta}{3 m_{p}^{2} H^{2} T^{4}}
$$

Let us assume that there is an interaction between ENCADE and pressureless cold dark matter and so the energy conservation equations are,

$$
\dot{\rho}_{x}+3 H\left(\rho_{x}+p_{x}\right)=-Q
$$

and

$$
\dot{\rho}_{m}+3 H \rho_{m}=Q
$$

where $Q=\Upsilon \rho_{x}$ and we choose $\Upsilon=3 b^{2} H\left(\frac{1+\Omega_{k}}{\Omega_{x}}\right)$ with $b$ is a coupling constant. So differentiating equation (4) w.r.t. 't' we get,

$$
\dot{\rho}_{x}=\frac{3 \chi H}{a} \sqrt{\frac{2 m_{p}^{2} \Omega_{x}^{2}}{3 n^{2} m_{p}^{2}+\gamma T^{-2} \ln \left(m_{p}^{2} T^{2}\right)+\beta T^{-2}}}
$$

where $\chi=-3 n^{2} m_{p}^{2} T^{-2}-2 \gamma T^{-4} \ln \left(m_{p}^{2} T^{2}\right)-2 \beta T^{-4}+\gamma T^{-4}$. Now $w_{e c n a d e}=\frac{p_{x}}{\rho_{x}}$ be the equation of state of ECNADE. So using the conservation equations we obtain the equation of state of ECNADE as,

$$
w_{\text {ecnade }}=-1-b^{2}\left(\frac{1+\Omega_{k}}{\Omega_{x}}\right)-\frac{2 \chi T^{2} \Omega_{x} \sqrt{3 m_{p}^{2}}}{3 a\left(3 n^{2} m_{p}^{2}+\gamma T^{-2} \ln \left(m_{p}^{2} T^{2}\right)+\beta T^{-2}\right)^{\frac{3}{2}}}
$$




\section{CORRESPONDENCE OF ECNADE MODEL WITH DBI-ESSENCE}

There have been many works aimed at connecting the string theory with inflation. While doing so, various ideas in string theory based on the concept of branes have proved themselves fruitful. One area which has been well explored in recent years, is inflation driven by the open string sector through dynamical Dp-branes. This is the so-called DBI (Dirac-Born-Infield) inflation, which lies in a special class of K-inflation models. Considering the dark energy scalar field is a Dirac-Born-Infeld (DBI) scalar field, the action of the field can be written as $[4-11]$

$$
d s_{d b i}=-\int d^{4} x a^{3}(t)\left[\digamma(\phi) \sqrt{1-\frac{\dot{\phi^{2}}}{\digamma(\phi)}}+V(\phi)-\digamma(\phi)\right]
$$

where $\digamma(\phi)$ is the tension and $V(\phi)$ is the potential. From the above expression, the corresponding pressure and the energy density of the scalar field becomes,

$$
p_{d b i}=\frac{\gamma-1}{\gamma} \digamma(\phi)-V(\phi) \quad \text { and } \quad \rho_{d b i}=(\gamma-1) \digamma(\phi)+V(\phi)
$$

where $\gamma$ is reminiscent from the usual relativistic Lorentz factor and is given by,

$$
\gamma=\left(1-\frac{\dot{\phi^{2}}}{\digamma(\phi)}\right)^{-\frac{1}{2}}
$$

Thus the equation of state is given by,

$$
w_{d b i}=\frac{\frac{\gamma-1}{\gamma} \digamma(\phi)-V(\phi)}{(\gamma-1) \digamma(\phi)+V(\phi)}=\frac{(\gamma-1) \digamma(\phi)-\gamma V(\phi)}{\gamma((\gamma-1) \digamma(\phi)+V(\phi))}
$$

Now we compare the equation of states and energy densities of the ECNADE and DBI-essence. Thus we get $w_{\text {ecnade }}=w_{d b i}$ and $\rho_{x}=\rho_{d b i}$ which gives,

$$
\frac{(\gamma-1) \digamma(\phi)-\gamma V(\phi)}{\gamma((\gamma-1) \digamma(\phi)+V(\phi))}=-1-b^{2}\left(\frac{1+\Omega_{k}}{\Omega_{x}}\right)-\frac{2 \chi}{3 a}\left(\frac{\sqrt{\frac{3 m_{p}^{2} \Omega_{x}^{2}}{3 n^{2} m_{p}^{2}+\gamma T^{-2} \ln \left(m_{p}^{2} T^{2}\right)+\beta T^{-2}}}}{3 n^{2} m_{p}^{2} T^{-2}+\gamma T^{-4} \ln \left(m_{p}^{2} T^{2}\right)+\beta T^{-4}}\right)
$$

and

$$
\frac{3 n^{2} m_{p}^{2}}{T^{2}}+\frac{\gamma \ln \left(m_{p}^{2} T^{2}\right)+\beta}{T^{4}}=(\gamma-1) \digamma(\phi)+V(\phi)
$$

So from (27), (29) and (30) we get the analytical expressions of $\digamma, \phi$ and $V$ as

$$
\begin{array}{r}
\digamma-\frac{1}{3 a\left(\gamma^{2}-1\right) T^{4} \Omega_{x}} \gamma\left[3 a b^{2}\left(\beta+3 m^{2} n^{2} T^{2}\right)\left(1+\Omega_{k}\right)+2 \sqrt{3}\left(\alpha-2 \beta-3 m^{2} n^{2} T^{2}\right) \Omega_{x}^{2} \sqrt{\frac{m^{2} T^{2}}{\beta+3 m^{2} n^{2} T^{2}+\alpha \ln \left[m^{2} T^{2}\right]}}\right. \\
+\alpha \ln \left[m^{2} T^{2}\right]\left(3 a b^{2}\left(1+\Omega_{k}\right)-4 \sqrt{3} \Omega_{x}^{2} \sqrt{\left.\left.\frac{m^{2} T^{2}}{\beta+3 m^{2} n^{2} T^{2}+\alpha \ln \left[m^{2} T^{2}\right]}\right)\right]} \frac{(31)}{3}\right) \\
\phi=\int\left[\frac { 1 } { 3 a \gamma T ^ { 4 } \Omega _ { x } } \left\{-3 a b^{2}\left(\beta+3 m^{2} n^{2} T^{2}\right)\left(1+\Omega_{k}\right)+2 \sqrt{3}\left(-\alpha+2 \beta+3 m^{2} n^{2} T^{2}\right) \Omega_{x}^{2} \sqrt{\frac{m^{2} T^{2}}{\beta+3 m^{2} n^{2} T^{2}+\alpha \ln \left[m^{2} T^{2}\right]}}\right.\right.
\end{array}
$$




$$
\left.\left.+\alpha \ln \left[m^{2} T^{2}\right]\left(-3 a b^{2}\left(1+\Omega_{k}\right)+4 \sqrt{3} \Omega_{x}^{2} \sqrt{\frac{m^{2} T^{2}}{\beta+3 m^{2} n^{2} T^{2}+\alpha \ln \left[m^{2} T^{2}\right]}}\right)\right\}\right]^{\frac{1}{2}} d t
$$

and

$$
\begin{gathered}
V=\frac{1}{3 a(\gamma+1) T^{4} \Omega_{x}}\left[3 a\left(\beta+3 m^{2} n^{2} T^{2}\right)\left(b^{2} \gamma\left(1+\Omega_{k}\right)+(1+\gamma) \Omega_{x}\right)+2 \sqrt{3}\left(\alpha-2 \beta-3 m^{2} n^{2} T^{2}\right) \Omega_{x}^{2}\right. \\
\left.\sqrt{\frac{m^{2} T^{2}}{\beta+3 m^{2} n^{2} T^{2}+\alpha \ln \left[m^{2} T^{2}\right]}}+\alpha \ln \left[m^{2} T^{2}\right]\left(3 a\left(b^{2} \gamma\left(1+\Omega_{k}\right)+(1+\gamma) \Omega_{x}\right)-4 \sqrt{3} \Omega_{x}^{2} \sqrt{\frac{m^{2} T^{2}}{\beta+3 m^{2} n^{2} T^{2}+\alpha \ln \left[m^{2} T^{2}\right]}}\right)\right]
\end{gathered}
$$

\section{CORRESPONDENCE OF ECNADE MODEL WITH YANG-MILLS DARK ENERGY}

Recent studies suggest that Yang-Mills field [12-16] can be considered as a useful candidate to describe the dark energy as in the normal scalar models the connection of field to particle physics models has not been clear so far and the weak energy condition cannot be violated by the field. In the effective Yang Mills Condensate (YMC) dark energy model, the effective Yang-Mills field Lagrangian is given by,

$$
\mathcal{L}_{Y M C}=\frac{1}{2} b F\left(\ln \left|\frac{F}{k^{2}}\right|-1\right)
$$

where $k$ is the re-normalization scale of dimension of squared mass, $F$ plays the role of the order parameter of the YMC where $F$ is given by, $F=-\frac{1}{2} F_{\mu \nu}^{a} F^{a \mu \nu}=E^{2}-B^{2}$. The pure electric case we have, $B=0 i . e . F=E^{2}$.

From the above Lagrangian we can derive the energy density and the pressure of the YMC in the flat FRW spacetime as

$$
\rho_{y}=\frac{1}{2}(y+1) b E^{2}
$$

and

$$
p_{y}=\frac{1}{6}(y-3) b E^{2}
$$

where $y$ is defined as,

$$
y=\ln \left|\frac{E^{2}}{k^{2}}\right|
$$

So the EoS of the YMC is given as,

$$
w_{y}=\frac{p_{y}}{\rho_{y}}=\frac{y-3}{3 y+3}
$$

Now we equate the EoS of the ECNADE and YMC i.e. $w_{y}=w_{E C N A D E}$ which gives,

$$
\frac{y-3}{3 y+3}=-1-b^{2}\left(\frac{1+\Omega_{k}}{\Omega_{x}}\right)-\frac{2 \chi}{3 a}\left(\frac{\sqrt{\frac{3 m_{p}^{2} \Omega_{x}^{2}}{3 n^{2} m_{p}^{2}+\gamma T^{-2} \ln \left(m_{p}^{2} T^{2}\right)+\beta T^{-2}}}}{3 n^{2} m_{p}^{2} T^{-2}+\gamma T^{-4} \ln \left(m_{p}^{2} T^{2}\right)+\beta T^{-4}}\right)
$$

which gives,

$$
y=\frac{-3 a b^{2}\left(\beta+3 m_{p}^{2} n^{2} T^{2}\right)\left(1+\Omega_{k}\right)-2 \sqrt{3}\left(\alpha-2 \beta-3 m_{p}^{2} n^{2} T^{2}\right) \Omega_{x}^{2} P+\alpha \ln \left[m_{p}^{2} T^{2}\right]\left(-3 a b^{2}\left(1+\Omega_{k}\right)+4 \sqrt{3} \Omega_{x}^{2} P\right)}{2 \sqrt{3} P \Omega_{x}^{2}\left(\alpha-2 \beta-3 m_{p}^{2} n^{2} T^{2}-2 \alpha \ln \left[m_{p}^{2} T^{2}\right]\right)+a\left(3 b^{2}\left(1+\Omega_{k}\right)+4 \Omega_{x}\right)\left(\beta+3 m_{p}^{2} n^{2} T^{2}+\alpha \ln \left[m_{p}^{2} T^{2}\right]\right)}
$$

where,

$$
P=\sqrt{\frac{m_{p}^{2} T^{2}}{\beta+3 m_{p}^{2} n^{2} T^{2}+\alpha \ln \left[m_{p}^{2} T^{2}\right]}}
$$




\section{CORRESPONDENCE OF ECNADE MODEL WITH CHAMELEON FIELD}

Many of the dark energy models might give a large correction to the Newton's law as the models are considered as a scalar field rolling down a flat potential. But in our solar system this scalar field becomes effectively massless. If this nearly massless scalar field existed on Earth it should have been detected in local test of equivalence principle and as a fifth force, unless the coupling to normal matter was unnatural from a theoretical standpoint. So a different approach is now being considered in general relativity, where the quintessence scalar field is allowed to interact non minimally with matter sector rather than the geometry and this interaction is introduced through an interference term in the action known as Chameleon scalar field [17-22].

The field equations of Hořava-Lifshitz gravity in presence of chameleon field $\phi$ can be written as,

$$
\begin{gathered}
H^{2}+\frac{k}{a^{2}}=\frac{\rho_{c} f(\phi)}{3 m_{p}^{2}}+\frac{k^{2}}{2 \Lambda a^{4}}+\frac{\Lambda}{2}+\frac{1}{2} \dot{\phi}^{2}+V(\phi)=\frac{f(\phi)}{3 m_{p}^{2}}\left(\rho_{m}+\rho_{X}\right)+\frac{k^{2}}{2 \Lambda a^{4}}+\frac{\Lambda}{2} \\
\dot{H}+\frac{3}{2} H^{2}+\frac{k}{2 a^{2}}=-\frac{p_{c} f(\phi)}{2 m_{p}^{2}}-\frac{k^{2}}{4 \Lambda a^{4}}+\frac{3 \Lambda}{2}-\frac{1}{2} \dot{\phi}^{2}+V(\phi)=-\frac{f(\phi)}{2 m_{p}^{2}}\left(p_{m}+p_{X}\right)-\frac{k^{2}}{4 \Lambda a^{4}}+\frac{3 \Lambda}{2}
\end{gathered}
$$

where $V(\phi)$ is the relevant potential and $f(\phi)$ is any analytical function of $\phi$. Also $\rho_{X}=\frac{3 m_{p}^{2}}{f(\phi)}\left[\frac{1}{2} \dot{\phi}^{2}+V(\phi)\right]$ and $p_{X}=\frac{2 m_{p}^{2}}{f(\phi)}\left[\frac{1}{2} \dot{\phi}^{2}-V(\phi)\right]$.

So the EoS of the Chameleon field is given as,

$$
w_{X}=\frac{p_{X}}{\rho_{X}}=\frac{2\left(\frac{1}{2} \dot{\phi}^{2}+V(\phi)\right)}{3\left(\frac{1}{2} \dot{\phi}^{2}-V(\phi)\right)}
$$

Now we equate the EoS and energy density of the Chameleon Field and ECNADE i.e. $w_{X}=w_{E C N A D E}$ and $\rho_{X}=\rho_{E C N A D E}$ which gives,

$$
\frac{2\left(\frac{1}{2} \dot{\phi}^{2}+V(\phi)\right)}{3\left(\frac{1}{2} \dot{\phi}^{2}-V(\phi)\right)}=-1-b^{2}\left(\frac{1+\Omega_{k}}{\Omega_{x}}\right)-\frac{2 \chi}{3 a}\left(\frac{\sqrt{\frac{3 m_{p}^{2} \Omega_{x}^{2}}{3 n^{2} m_{p}^{2}+\gamma T^{-2} \ln \left(m_{p}^{2} T^{2}\right)+\beta T^{-2}}}}{3 n^{2} m_{p}^{2} T^{-2}+\gamma T^{-4} \ln \left(m_{p}^{2} T^{2}\right)+\beta T^{-4}}\right)
$$

and

$$
\frac{3 m_{p}^{2}}{f(\phi)}\left[\frac{1}{2} \dot{\phi}^{2}+V(\phi)\right]=\frac{3 n^{2} m_{p}^{2}}{T^{2}}+\frac{\gamma \ln \left(m_{p}^{2} T^{2}\right)+\beta}{T^{4}}
$$

Solving the above two equations we get the expressions of $f$ and $V$ as,

$f=-\frac{6 a m^{2} T^{4} \dot{\phi}^{2} \Omega_{X}}{\left(a\left(\beta+3 m_{p}^{2} n^{2} T^{2}\right)\left(3 b^{2}\left(1+\Omega_{k}\right)+\Omega_{X}\right)+2 \sqrt{3}\left(\alpha-2 \beta-3 m_{p}^{2} n^{2} T^{2}\right) \Omega_{x}^{2} P+\alpha \ln \left[m_{p}^{2} T^{2}\right]\left(a\left(3 b^{2}\left(1+\Omega_{k}\right)+\Omega_{X}\right)-4 \sqrt{3} \Omega_{x}^{2} P\right)\right)}$

and

$V=-\frac{\dot{\phi}^{2}\left(\left(a\left(\beta+3 m_{p}^{2} n^{2} T^{2}\right)\left(3 b^{2}\left(1+\Omega_{k}\right)+5 \Omega_{X}\right)+2 \sqrt{3}\left(\alpha-2 \beta-3 m_{p}^{2} n^{2} T^{2}\right) \Omega_{x}^{2} P+\alpha \ln \left[m_{p}^{2} T^{2}\right]\left(a\left(3 b^{2}\left(1+\Omega_{k}\right)+5 \Omega_{X}\right)-4 \sqrt{3} \Omega_{x}^{2} P\right)\right)\right)}{2\left(a\left(\beta+3 m_{p}^{2} n^{2} T^{2}\right)\left(3 b^{2}\left(1+\Omega_{k}\right)+\Omega_{X}\right)+2 \sqrt{3}\left(\alpha-2 \beta-3 m_{p}^{2} n^{2} T^{2}\right) \Omega_{x}^{2} P+\alpha \ln \left[m_{p}^{2} T^{2}\right]\left(a\left(3 b^{2}\left(1+\Omega_{k}\right)+\Omega_{X}\right)-4 \sqrt{3} \Omega_{x}^{2} P\right)\right)}$

where,

$$
P=\sqrt{\frac{m_{p}^{2} T^{2}}{\beta+3 m_{p}^{2} n^{2} T^{2}+\alpha \ln \left[m_{p}^{2} T^{2}\right]}}
$$




\section{CORRESPONDENCE OF ECNADE MODEL WITH NON-LINEAR ELECTRODYNAMICS FIELD}

Recently a new approach has been taken to avoid the cosmic singularity through a nonlinear extension of the Maxwell electromagnetic theory. Another interesting feature can be viewed that an exact regular black hole solution has been recently obtained proposing Einstein-dual nonlinear electrodynamics. Exact solutions of the Einstein's field equations coupled with nonlinear electrodynamics (NLED) reveal an acceptable nonlinear effect in strong gravitational and magnetic fields. Also the General Relativity (GR) coupled with NLED effects can explain the primordial inflation.

The Lagrangian density for free fields in the Maxwell electrodynamics may be written as [23-25],

$$
\mathcal{L}_{\mathcal{M}}=-\frac{1}{4 \mu} F^{\mu \nu} F_{\mu \nu}
$$

where $F^{\mu \nu}$ is the electromagnetic field strength tensor and $\mu$ is the magnetic permeability.

Here we consider the generalization of Maxwell electro-magnetic Lagrangian up to the second order terms of the fields as

$$
\mathcal{L}=-\frac{1}{4 \mu_{0}} F+\omega F^{2}+\eta F^{* 2}
$$

where $\omega$ and $\eta$ are arbitrary constants,

$$
F^{*} \equiv F_{\mu \nu}^{*} F^{\mu \nu}
$$

and $F_{\mu \nu}^{*}$ is the dual of $F_{\mu \nu}$. Now we consider the homogeneous electric field $E$ in plasma gives rise to an electric current of charged particles and then rapidly decays. So the squared magnetic field $B^{2}$ dominates over $E^{2}$, i.e., in this case, $E^{2} \approx 0$ and hence $F=2 B^{2}$. So $F$ is now only the function of magnetic field.

Now the pressure and energy density of the Non-linear Electrodynamics Field is,

$$
p_{N E}=\frac{B^{2}}{6 \mu}\left(1-40 \mu \omega B^{2}\right)
$$

and

$$
\rho_{N E}=\frac{B^{2}}{2 \mu}\left(1-8 \mu \omega B^{2}\right)
$$

The weak condition $(\rho>0)$ is obeyed for, $B<\frac{1}{2 \sqrt{2 \mu \omega}}$ and pressure will be negative when $B>\frac{1}{2 \sqrt{10 \mu \omega}}$. The magnetic field generates dark energy if strong energy condition is violated i.e., $\rho_{B}+3 p_{B}<0$, i.e., if $B>\frac{1}{2 \sqrt{6 \mu \omega}}$.

So the EoS of the Nonlinear Electrodynamics Field is given as,

$$
w_{N E}=\frac{p_{N E}}{\rho_{N E}}=\frac{\frac{B^{2}}{6 \mu}\left(1-40 \mu \omega B^{2}\right)}{\frac{B^{2}}{2 \mu}\left(1-8 \mu \omega B^{2}\right)}=\frac{1-40 \mu \omega B^{2}}{3\left(1-8 \mu \omega B^{2}\right)}
$$

Now we equate the EoS of the Nonlinear Electrodynamics Field and ECNADE i.e. $w_{N E}=w_{E C N A D E}$ gives,

$$
\frac{\left(1-40 \mu \omega B^{2}\right)}{3\left(1-8 \mu \omega B^{2}\right)}=-1-b^{2}\left(\frac{1+\Omega_{k}}{\Omega_{x}}\right)-\frac{2 \chi}{3 a}\left(\frac{\sqrt{\frac{3 m_{p}^{2} \Omega_{x}^{2}}{3 n^{2} m_{p}^{2}+\gamma T^{-2} \ln \left(m_{p}^{2} T^{2}\right)+\beta T^{-2}}}}{3 n^{2} m_{p}^{2} T^{-2}+\gamma T^{-4} \ln \left(m_{p}^{2} T^{2}\right)+\beta T^{-4}}\right)
$$


From where we get the expression of magnetic field as,

$B=\sqrt{\frac{a\left(\beta+3 m_{p}^{2} n^{2} T^{2}\right)\left(3 b^{2}\left(1+\Omega_{k}\right)+4 \Omega_{X}\right)+2 \sqrt{3}\left(\alpha-2 \beta-3 m_{p}^{2} n^{2} T^{2}\right) \Omega_{x}^{2} P+\alpha \ln \left[m_{p}^{2} T^{2}\right]\left(a\left(3 b^{2}\left(1+\Omega_{k}\right)+4 \Omega_{X}\right)-4 \sqrt{3} \Omega_{x}^{2} P\right)}{8 \mu \omega\left(a\left(\beta+3 m_{p}^{2} n^{2} T^{2}\right)\left(3 b^{2}\left(1+\Omega_{k}\right)+8 \Omega_{X}\right)+2 \sqrt{3}\left(\alpha-2 \beta-3 m_{p}^{2} n^{2} T^{2}\right) \Omega_{x}^{2} P+\alpha \ln \left[m_{p}^{2} T^{2}\right]\left(a\left(3 b^{2}\left(1+\Omega_{k}\right)+8 \Omega_{X}\right)-4 \sqrt{3} \Omega_{x}^{2} P\right)\right)}}$

where,

$$
P=\sqrt{\frac{m_{p}^{2} T^{2}}{\beta+3 m_{p}^{2} n^{2} T^{2}+\alpha \ln \left[m_{p}^{2} T^{2}\right]}}
$$

\section{CORRESPONDENCE OF ECNADE MODEL WITH HESSENCE DARK ENERGY}

Wei et al [26] proposed a novel non-canonical complex scalar field named "hessence" which play the role of quintom. In the hessence model the so called internal motion $\dot{\theta}$ where $\theta$ is the internal degree of freedom of hessence plays a Phantom like role and the Phantom divide transitions is also possible.

Now the pressure and energy density of the hessence dark energy is given by [27, 28]

$$
p_{X}=\frac{1}{2}\left(\dot{\phi}^{2}-\phi^{2} \dot{\theta}^{2}\right)-V(\phi)
$$

and

$$
\rho_{X}=\frac{1}{2}\left(\dot{\phi}^{2}-\phi^{2} \dot{\theta}^{2}\right)+V(\phi)
$$

where $Q=a^{3} \phi^{2} \dot{\theta}=$ constant is the total conserved charge. So the EoS of the is given as,

$$
w_{X}=\frac{p_{X}}{\rho_{X}}=\frac{\frac{1}{2}\left(\dot{\phi}^{2}-\frac{Q^{2}}{a^{6} \phi^{2}}\right)-V(\phi)}{\frac{1}{2}\left(\dot{\phi}^{2}-\frac{Q^{2}}{a^{6} \phi^{2}}\right)+V(\phi)}
$$

Now we equate the EoS and energy density of the and ECNADE i.e. $w_{X}=w_{E C N A D E}$ and $\rho_{X}=\rho_{E C N A D E}$ which gives,

$$
\frac{p_{X}}{\rho_{X}}=\frac{\frac{1}{2}\left(\dot{\phi}^{2}-\frac{Q^{2}}{a^{6} \phi^{2}}\right)-V(\phi)}{\frac{1}{2}\left(\dot{\phi}^{2}-\frac{Q^{2}}{a^{6} \phi^{2}}\right)+V(\phi)}=-1-b^{2}\left(\frac{1+\Omega_{k}}{\Omega_{x}}\right)-\frac{2 \chi}{3 a}\left(\frac{\sqrt{\frac{3 m_{p}^{2} \Omega_{x}^{2}}{3 n^{2} m_{p}^{2}+\gamma T^{-2} \ln \left(m_{p}^{2} T^{2}\right)+\beta T^{-2}}}}{3 n^{2} m_{p}^{2} T^{-2}+\gamma T^{-4} \ln \left(m_{p}^{2} T^{2}\right)+\beta T^{-4}}\right)
$$

and

$$
\frac{1}{2}\left(\dot{\phi}^{2}-\frac{Q^{2}}{a^{6} \phi^{2}}\right)+V(\phi)=\frac{3 n^{2} m_{p}^{2}}{T^{2}}+\frac{\gamma \ln \left(m_{p}^{2} T^{2}\right)+\beta}{T^{4}}
$$

which gives, $V(\phi)=\frac{1}{6 a T^{4} \Omega_{X}}\left[3 a\left(\beta+3 m_{p}^{2} n^{2} T^{2}\right)\left(b^{2}\left(1+\Omega_{k}\right)+2 \Omega_{X}\right)+2 \sqrt{3}\left(\alpha-2 \beta-3 m_{p}^{2} n^{2} T^{2}\right) \Omega_{X}^{2} P\right.$

$$
\left.+\alpha \ln \left[m_{p}^{2} T^{2}\right]\left(3 a\left(b^{2}\left(1+\Omega_{k}\right)+2 \Omega_{X}\right)-4 \sqrt{3} \Omega_{X}^{2} P\right)\right]
$$

and

$$
\begin{aligned}
& \phi=\int \frac{1}{\sqrt{3}}\left[\sqrt { \frac { 1 } { a ^ { 6 } T ^ { 4 } \phi ^ { 2 } \Omega _ { X } } } \left[\sqrt{-3 a^{6} b^{2}\left(\beta+3 m_{p}^{2} n^{2} T^{2}\right) \phi^{2}\left(1+\Omega_{k}\right)+3 Q^{2} T^{4} \Omega_{X}}\right.\right. \\
& \left.\left.+\overline{2 \sqrt{3} a^{5}\left(-\alpha+2 \beta+3 m_{p}^{2} n^{2} T^{2}\right) \phi^{2} \Omega_{X}^{2} P+a^{5} \alpha \phi^{2} \ln \left[m_{p}^{2} T^{2}\right]\left(-3 a b^{2}\left(1+\Omega_{k}\right)+4 \sqrt{3} \Omega_{X}^{2} P\right)}\right]\right] d t
\end{aligned}
$$

where,

$$
P=\sqrt{\frac{m_{p}^{2} T^{2}}{\beta+3 m_{p}^{2} n^{2} T^{2}+\alpha \ln \left[m_{p}^{2} T^{2}\right]}}
$$




\section{DISCUSSIONS}

Several authors have extensively discussed about ECHDE [52-55] and ECNADE [56-59] model to explain recent acceleration in different context using different dark energy models. In this work, we have considered the entropy-corrected new agegraphic dark energy (ECNADE) model is applied in a universe governed by a modified gravity model named Hořava-Lifshitz gravity in FRW universe to understand the behaviour of the potentials when a correspondence between the ECNADE and other dark energies have been considered. Since the new agegraphic dark energy (NADE) model in the framework of quantum gravity is being used as a source to probe the nature of dark energy, so we have used the logarithmic corrected version of NADE which is usually motivated from the important feature of LQG to understand tghe nature of the universe in very early epoch. The interaction between ECNADE and dark matter have been considered. Choosing some particular form of interaction term, we have derived the equation of state for ECNADE. Recent studies reveals that the scalar field models are very effective as a candidate of dark energy, so we use these models according to the evolutionary behavior of the interacting ECNADE. So we compare the equation of state of different scalar field models with the ECNADE. We have discussed the correspondence between ECNADE and other dark energy models such as DBI-essence, Yang-Mills dark energy, Chameleon field, Non-linear electrodynamics field

and hessence dark energy in the context of Horava-Lifshitz gravity and reconstructed the potentials and the dynamics of the scalar field theory which describe the ECNADE.

\section{Acknowledgement:}

The authors are thankful to IUCAA, Pune, India for warm hospitality where part of the work was carried out.

\section{References:}

[1] A. G. Riess et al, Astron. J. 1161009 (1998).

[2] D. N. Spergel Astrophys. J. Suppl. 148175 (2003).

[3] S. Perlmutter et al, Astrophys. J. 517565 (1999).

[4] J. E. Lidsey and I. Huston, JCAP 0707002 (2007).

[5] W. H. Kinney and K. Tzirakis, Phys. Rev. D 77103517 (2008).

[6] J. Martin and M. Yamaguchi, Phys. Rev. D 77123508 (2008).

[7] M. Spaliński, JCAP 01705 (2007); Phys. Lett. B 650313 (2007).

[8] X. Chen, M. X. Huang, S. Kachru and G. Shiu, JCAP 0701002 (2007).

[9] S. Kecskemeti, J. Maiden, G. Shiu and B. Underwood, JHEP 0609076 (2006).

[10] M. X. Huang, G. Shiu and B. Underwood, Phys. Rev. D 77023511 (2008).

[11] D. Seery and J. E. Lidsey, Phys. Rev. D 75043505 (2007).

[12] Y. Zhang, T. Y. Xia and W. Zhao, Class. Quant. Grav. 243309 (2007).

[13] T. Y. Xia and Y. Zhang, Phys. Lett. B 65619 (2007).

[14] M. Tong, Y. Zhang and T. Xia, Int. J. Mod. Phys. D 18797 (2009).

[15] W. Zhao, Int. J. Mod. Phys. D17 1245 (2008); W. Zhao and Y. Zhang, Class. Quant. Grav. 233405 (2006); W. Zhao, Astron. Astrophys. 9874 (2009); W. Zhao and D. Xu, Int. J. Mod. Phys. D 161735 (2007); 
Y. Zhang, T. Y. Xia, and W. Zhao, Class. Quant. Grav.24 3309 (2007).

[16] Z. Yang, Chin. Phys. Lett. 211183 (2004).

[17] A. -C. Davis, C. A. O. Schelpe and D. J. Shaw, Phys. Rev. D 80064016 (2009).

[18] S. Chakraborty and U. Debnath, IJMPA 254691 (2010).

[19] N. Banerjee et al, Pramana 74 L481 (2010); N. Banerjee et al, Phys. Rev. D 78043512 (2008).

[20] Ph. Brax, arXiv:0410103 [astro-ph] (2004); Proceedings of the "Phi in the Sky" conference "The Quest for Cosmological Scalar Fields", doi:10.1063/1.1835177, Vol. 736, p. 105-110, 8-10 July 2004, Porto, Portugual.

[21] H. Wei and R. -G. Cai, Phys. Rev. D71 043504 (2005).

[22] D. F. Mota and D. J. Shaw, Phys. Rev. Lett. 97151102 (2006).

[23] R. Garca-Salcedo et al, arXiv:1006.2276 [gr-qc] (2010).

[24] V. A. De Lorenci et al, Phys. Rev. D 65063501 (2002).

[25] C. S. Camara et al, Phys. Rev. D 69103504 (2004).

[26] H. Wei, R. -G. Cai and D. F. Zhang, Class. Quant. Grav. 223189 (2005); H. Wei and S. N. Zhang, Phys. Rev. D76 063005 (2007); H. Wei, N. Tang and S. N. Zhang, Phys. Rev. D 75043009 (2007).

[27] M. Alimohammadi and H. M. Sadjadi, Phys. Rev. D73 083527 (2006).

[28] S. Chakraborty and U. Debnath, Int. J. Mod. Phys. D 192071 (2010).

[29] S. Nojiri and S. D. Odintsov Int. J. Geom. Meth. Mod. Phys. 4115 (2007).

[30] S. Nojiri and S. D. Odintsov, arXiv: 0807.0685 [hep-th] (2008); Problems of Modern Theoretical Physics, A Volume in honour of Prof. I. L. Buchbinder in the occasion of his 60th birthday, p.266-285, TSPU Publishing, Tomsk.

[31] P. Hořava, JHEP 0903020 (2009).

[32] P. Hořava, Phys. Rev. D 79084008 (2009).

[33] P. Hořava, Phys. Rev. Lett. 102161301 (2009).

[34] P. Hořava, Phys. Lett. B 694172 (2010).

[35] E. M. Lifshitz, Zh. Eksp. Teor. Fiz. 11255 (1949).

[36] R. G. Cai et al, Phys. Rev. D 80041501 (2009); M. R. Setare and M. Jamil, JCAP 02010 (2010).

[37] G. Calcagni, JHEP 0909112 (2009).

[38] H. Lu et al, Phys. Rev. Lett. 103091301 (2009).

[39] G. t Hooft, 9310026 [gr-qc] (2009); L. Susskind, J. Math. Phys. 366377 (1995).

[40] S. W. Hawking, Commun. Math. Phys. 43199 (1975); S. W. Hawking, Phys. Rev. D 13191 (1976); J. D. Bekenstein, Phys. Rev. D 23287 (1981). 
[41] A. Cohen, D. Kaplan and A. Nelson, Phys. Rev. Lett. 824971 (1999).

[42] P. Hořava and D. Minic, Phys. Rev. Lett. 851610 (2000); S. D. Thomas, Phys. Rev. Lett. 89081301 (2002).

[43] S. D. H. Hsu, Phys. Lett. B 594, 13 (2004).

[44] K. A. Meissner, Class. Quantum Grav. 215245 (2004).

[45] H. Wei, Commun. Theor. Phys. 52743 (2009).

[46] R. -G. Cai, Phys. Lett. B 657228 (2007).

[47] H. Wei and R. -G. Cai, Phys. Lett. B 660113 (2008).

[48] F. Karolyhazy, Nuovo. Cim. A 42390 (1966).

[49] M. Maziashvili, Int. J. Mod. Phys. D 161531 (2007); M. Maziashvili, Phys. Lett. B 652165 (2007).

[50] H. Wei, Commun. Theor. Phys. 52743 (2009); M. Jamil and M. U. Farooq, JCAP 03001 (2010).

[51] R. L. Arnowitt, S. Deser and C. W. Misner, Gravitation: an introduction to current research", Louis Witten ed. (Wiley 1962), chapter 7, pp 227-265 (2004).

[52] W. Hao, Commun. Theor. Phys. 52743 (2009).

[53] M. R. Setare and M. Jamil, Europhys. Lett. 9249003 (2010).

[54] A. K. Mohammadi, and M. Malekjani, arXiv: 1004.1720v2 [gr-qc] (2011).

[55] E. Ebrahimi and A. Sheykhi, arXiv: 1011.5005v2 [hep-th] (2011).

[56] M. Jamil and A. Sheykhi, Int. J. Theor. Phys. 50625 (2011).

[57] M. Malekjani and A. K. Mohammadi, arXiv: 1004.1017v2 [gr-qc] (2010).

[58] K. Karami et al, Europhys. Lett. 9369001 (2011); K. Karami et al, Gen. Rel. Grav. 4327 (2011); K. Karami and A. Sorouri, Phys. Scr. 82025901 (2010).

[59] M. U. Farooq, M. Jamil and M. A. Rashid, Int. J. Theor. Phys. 492278 (2010). 